\title{
Figure géométrique et construction philosophique chez Pascal
}

Jean Mesnard

\section{(2) OpenEdition \\ Journals}

Édition électronique

URL : http://journals.openedition.org/ccibp/288

DOI : $10.4000 /$ ccibp. 288

ISSN : 2493-7460

Éditeur

Centre international Blaise Pascal

\section{Édition imprimée}

Date de publication : 1 décembre 2011

Pagination : 4-13

ISBN : 978-2-84516-551-9

ISSN : 0249-6674

Référence électronique

Jean Mesnard, «Figure géométrique et construction philosophique chez Pascal », Courrier du Centre international Blaise Pascal [En ligne], 33 | 2011, mis en ligne le 19 novembre 2015, consulté le 09 octobre 2020. URL : http://journals.openedition.org/ccibp/288; DOI : https://doi.org/10.4000/ccibp. 288

Ce document a été généré automatiquement le 9 octobre 2020.

Centre international Blaise Pascal 


\title{
Figure géométrique et construction philosophique chez Pascal
}

\author{
Jean Mesnard
}

1 Quelle idée Pascal se faisait-il de la géométrie ? En quoi consistait pour lui son apport particulier au domaine des sciences? En allant plus loin, quel fruit pouvait-on en espérer pour l'économie générale de la connaissance? Voilà des questions que Pascal s'est indiscutablement posées. Sa réponse peut être cherchée d'abord dans quelques textes théoriques, dans les éléments d'une sorte de philosophie de la géométrie à laquelle il s'est assez souvent livré. Nous procéderons brièvement à une enquête en ce sens. Mais il convient aussi de s'interroger sur sa pratique personnelle de la géométrie et, ce qui est plus délicat, sur le recours implicite fait à cette science dans des réflexions, éventuellement philosophiques, dont l'objet la déborde. Sur ce dernier point, il suffira d'analyser quelques exemples significatifs. Nous retiendrons particulièrement des cas où la figure géométrique apparaît comme en filigrane sous l'emploi du discours philosophique.

2 Ce qui frappe dans les réflexions théoriques auxquelles nous devons d'abord nous arrêter, c'est à la fois le souci d'atteindre une sorte d'essence de la géométrie, au cœur de toutes ses applications imaginables, et le sentiment de l'extraordinaire extension du champ qu'elle peut couvrir, lui permettant notamment de franchir sans peine les limites du concret, où elle est proprement chez elle, pour saisir des notions qui sont du ressort de l'esprit. En somme, il existe un rapport fondamental entre la géométrie et la logique.

\section{La géométrie selon Pascal : figures et proportions}

3 Commençons par un texte qui a jusqu'ici peu attiré l'attention, sans doute parce qu'il n'émane pas directement de Pascal, mais de sa biographe, sa sœur Gilberte. On sait, selon cette dernière, comment celui qui n'était encore qu'un enfant réussit à imposer à ses proches une précoce vocation aux mathématiques, contre laquelle ils résistaient, en obtenant de son père, savant en cette science et probablement auteur malgré lui de 
cette vocation, une définition qui se révéla suffisante pour entrainer des recherches révélatrices de dons exceptionnels. Quelle fut donc cette définition si lumineuse et si féconde? Elle est brève, mais dense. Devant le refus obstiné de son père de lui enseigner sans tarder "la mathématique", l'enfant, peut-être par impatience, et toujours selon Gilberte,

lui demanda un jour ce que c'était que cette science, et de quoi on y traitait. Mon père, poursuit-elle, lui dit en général que c'était le moyen de faire des figures justes, et de trouver les proportions qu'elles avaient entre elles.

La formulation est très rigoureuse ; mais elle appelle quelques mots de commentaire. Que faut-il entendre par "figures justes »? Notons d'abord que «la mathématique » dont il est question ici s'identifie en fait avec la géométrie : conséquence, à la fois d'une hiérarchisation des disciplines qui aboutirait à ne reconnaître qu'un second rang à l'arithmétique; et surtout du fait que tout peut entrer dans la géométrie, puisque les nombres peuvent eux-mêmes recevoir une disposition spatiale, et devenir figures, par exemple lorsqu'on parle de carrés et de cubes; et que toute figure appelle mesure, c'est-à-dire nombre, ce qui n'est pas aussi vrai dans l'autre sens. Ajoutons que, si le champ de la géométrie couvre la grandeur continue et celui de l'arithmétique la grandeur discontinue, la discontinuité se manifeste très aisément en géométrie, alors que la continuité ne peut être qu'approchée en arithmétique. Cette primauté de la géométrie est peut-être moins sensible aujourd'hui : effet possible de la critique portée à l'époque moderne contre la notion de qualité, si souvent invoquée par la science médiévale, surtout en physique, et de la tendance à faire de la quantification le signe de la modernité. Mais ce n'est pas exactement ce problème qui peut nous retenir ici. En fait, ce qui fait de la figure un élément capital de la géométrie, c'est qu'elle est forme, comme il ressort de son nom. Elle s'adresse à la fois aux yeux et à l'esprit. Elle est signe en même temps que sens, ce qui enrichit son contenu expressif. Par là même, la géométrie semble constituer la partie la plus compréhensive des mathématiques.

5 Pour essayer de définir l'expression « figures justes ", nous sommes conduits à préciser encore les caractères de la géométrie. Sans doute faut-il d'abord songer au travail concret du géomètre arpenteur, chargé de reproduire graphiquement le dessin et les dimensions d'un terrain, avec, pour finir, mesure fournie par des nombres, qui met en jeu l'arithmétique, et, par là même, donne accès à l'ensemble de la mathématique. Une même réflexion sur la géométrie pratique permet de comprendre ce qu'elle implique en matière de saisie de proportions. D'abord, entre le terrain et l'épure qui en est transcrite une proportion est déjà posée par l'échelle adoptée. De là découle d'ailleurs une possibilité de mesure, qui est déjà établissement d'une proportion. D'un autre côté, la comparaison entre figures diverses introduit à des analyses beaucoup plus nombreuses. Certains cas particuliers sont d'un extrême intérêt à cet égard. Ainsi celui de la similitude d'une figure à une autre, qui conserve les proportions internes, communes à toutes les figures d'une même série, tout en modifiant les proportions externes, qui s'appliquent de l'une à l'autre. S'ensuivent diverses notions issues de la géométrie, mais à portée beaucoup plus vaste, comme celles de figure (en un autre sens, très pascalien), ou de modèle (terme également pascalien).

6 À côté du rôle ainsi dévolu à la ressemblance, il convient d'accorder une place à celui du changement, du moins à certaines de ses modalités, où se conjuguent ressemblances et différences. Ainsi existe-t-il un certain jeu sur les proportions dans la méthode, si soigneusement retenue par Leibniz, qui permet de concevoir une "génération des 
sections coniques ", grâce à laquelle toutes les sections de cône, cercle, ellipse, parabole, hyperbole, et même, plus simplement encore, point, droite et angle, sont successivement engendrées par le mouvement d'un plan coupant un cône. Il est clair qu'à chaque étape de ce mouvement, des proportions nouvelles s'établissent entre les principaux éléments des figures ainsi formées, le cercle générateur, d'une part, les sections de cône plus complexes, de l'autre. Il faudra y revenir.

Toutes ces opérations ont une portée générale. Elles font ressortir ce qui est peut-être un caractère encore plus fondamental de la géométrie, et qui traduit de nouveau son essence formelle, celui qui la conduit à se fonder sur la considération de ces schémas que sont les figures. Schémas qui sont création idéale de l'esprit, et tenus pour tels dans des raisonnements où ils sont les véritables objets, abstraction faite de leur rapport incontestable avec des objets réels, mais moins parfaits, dont ils sont l'image plus pure. C'est la pureté de cette image qui commande son efficacité. C'est elle aussi qui en fait un modèle, susceptible d'application à tout objet offrant un système comparable de proportions, fût-il imparfait.

\section{La géométrie selon Pascal : L'espace et ses problèmes}

8 La seconde réflexion théorique à laquelle il convient de nous arrêter est encore plus essentielle en ce qu'elle émane de Pascal lui-même. Elle ne nous a pas été toutefois transmise directement par son auteur, mais par une copie de Leibniz, qui l'a sans doute quelque peu glosée. Inversement, les gloses éventuelles du philosophe allemand ont pu lui être inspirées par le texte original qu'il avait sous les yeux, qu'il contribuerait donc encore ainsi à faire connaître. Quoi qu'il en soit, la parenté entre les deux esprits est telle sur ce point, comme il en est d'autres preuves, que nous accédons sûrement par ce texte à l'authentique pensée de Pascal, dont l'antériorité, en tout état de cause, ne peut faire aucun doute.

9 Ce texte est un fragment d'Introduction à la géométrie, probablement contemporain des Nouveaux Éléments que commençait à préparer le grand Arnauld pour l'enseignement de cette science, à une date qui pourrait être 1655 . Il comporte d'abord un passage qu'il y a tout lieu de considérer comme une copie fidèle, d'autant qu'il rend un son nettement pascalien. Il propose une définition originale de la géométrie :

L'objet de la pure géométrie est l'espace, dont elle considère la triple étendue en trois sens divers qu'on appelle dimensions, lesquelles on distingue par les noms de longueur, largeur et profondeur, en donnant indifféremment chacun de ces noms à chacune de ces dimensions, pourvu qu'on ne donne pas le même à deux ensemble. Elle suppose que tous ces termes-là sont connus d'eux-mêmes.

Deux traits dans ce passage conduisent nécessairement à Pascal. D'abord le souci de la « pure géométrie » et la distinction radicale, déduite des recherches de physique sur le vide et résolument anti-cartésienne, entre l'espace et les corps qui l'occupent. Nouvelle manière d'insister sur le caractère formel de la géométrie, qui entraîne d'ailleurs une conception également formelle des corps, eux aussi réduits à leurs formes, et donc susceptibles de signifier plusieurs objets. Fait aussi reconnaître l'auteur de L'Esprit géométrique l'affirmation que les termes désignant les dimensions « sont connus d'euxmêmes ", c'est-à-dire n'ont pas besoin de définitions, comme tous ceux que la nature suffit à procurer à l'esprit, et ne sont donc pas sujets à ambiguïté. 
11 Entre les points particuliers qu'aborde ensuite l'écrit copié par Leibniz, il est une notation qui revient à plusieurs reprises et qui témoigne encore d'une originalité significative. C'est l'énoncé des diverses manières dont deux éléments de l'espace, en somme deux figures, à commencer par le point et la ligne, les points et les lignes, peuvent différer. À côté de caractéristiques assez banales, d'abord évidemment l'égalité, puis la longueur et la largeur, une attention particulière est apportée à la situation, et une autre à la direction. Ainsi, à la pure géométrie succède une amorce de mécanique. Dans un cas comme dans l'autre, il s'agit d'établir des rapports, lesquels, par égalité ou inégalité entre eux, conduisent aux proportions, support de la connaissance. Ainsi se rejoignent les diverses voies d'accès vers la géométrie suivies par Pascal.

Dans les notes personnelles, suggérées sans doute un peu par l'écrit qu'il découvrait, Leibniz enrichit l'arsenal de définitions qu'il y a trouvées. Il n'en change pas l'esprit, mais apporte quelques précisions complémentaires. Lui aussi distingue l'espace du corps, en ajoutant : «L'espace est une chose étendue et rien davantage. Un corps est une chose étendue, capable d'agir. Agir est être cause d'un changement. » Ainsi le corps introduit une possibilité de mouvement qui invite ainsi à le considérer comme une cause. D'où nouvelle définition, sans doute d'après la scolastique : « Cause est une chose prise dans un certain état dans lequel elle ne peut être quand une autre arrive, et peut être entendue parfaitement avant l'autre. L'autre s'appelle l'effet. » La relation causeeffet, qui a fait l'objet de critiques sévères de la part de nombreux savants modernes, se trouve ainsi mise en exergue, aussi bien par Pascal que par Leibniz. À notre avis, c'est moins le principe de la relation qui appelle réserves que la rigueur variable avec laquelle elle est exploitée. Nous en découvrirons bientôt un exemple très remarquable. Une autre relation retient beaucoup l'attention des deux philosophes : c'est celle qui s'établit entre le tout et les parties: elle rejoint un peu celle de diverses positions entre objets.

13 Toutes ces réflexions suggérées par notre second texte ne font que souligner, comme on l'a déjà indiqué au passage, le caractère formel de la géométrie et la pratique de la schématisation qu'elle comporte.

\section{La géométrie selon Pascal : inutile en elle-même, mais modèle du savoir}

14 Nous relèverons encore un troisième texte, cette fois très directement pascalien, et dont l'exégèse peut se faire assez simplement. Il est postérieur aux précédents, mais se place très facilement dans leur suite. Il appartient à une lettre datée du 19 août 1660 et adressée au grand mathématicien toulousain Fermat, qui avait invité Pascal, alors en séjour dans sa ville natale de Clermont-Ferrand, à venir le rencontrer dans un lieu choisi à mi-chemin de leurs deux demeures respectives. La réponse fut un refus courtois, motivé par des raisons de santé, mais surtout par l'effet d'une sorte de distance prise avec la pratique de la géométrie. Voici ses termes :

... Pour vous parler franchement de la géométrie, je la trouve le plus haut exercice de l'esprit; mais en même temps je la connais pour si inutile que je fais peu de différence entre un homme qui n'est que géomètre et un habile artisan. Aussi je l'appelle le plus beau métier du monde ; mais enfin ce n'est qu'un métier, et j'ai dit 
souvent qu'elle n'est bonne que pour faire l'essai, mais non pas l'emploi de notre force... n'aura pas de nouveau prolongement. Mais s'il se refuse désormais à poursuivre cette œuvre comme une spécialité, il continue à y voir ce qui peut donner « force » à notre esprit et lui permettre de s'employer efficacement dans son usage général, où ses lois demeurent souveraines. C'est ainsi que toute la réflexion du philosophe, toute la pensée sur l'homme peuvent en rester pénétrées. La justesse des figures introduit à la justesse de l'esprit.

\section{Figures internes aux mathématiques}

16 Il nous appartient maintenant de nous étendre sur ces applications de la géométrie. Mais, avant même d'entrer dans le domaine philosophique, il est intéressant, dans la constitution de la géométrie elle-même, dans la logique où elle se généralise, et dans les ressources qu'elle offre à des disciplines qui lui font directement appel, arithmétique, mécanique ou physique d'abord, d'essayer de saisir de multiples rapports entre procédures semblables, dont le déroulement s'opère selon des figures qui peuvent se superposer. La géométrie, et diverses sciences qui relèvent d'elle, pourraient alors se présenter comme d'amples collections de figures, susceptibles de se trouver imbriquées les unes dans les autres.

Il peut arriver que des superpositions de figures, ou quasi-figures, puissent se réaliser entre disciplines mathématiques différentes. C'est bien ce que constate Pascal dans le rapport entre géométrie et arithmétique :

Les nombres imitent l'espace, qui sont de nature si différente.

En effet, de la discontinuité, propriété des nombres, à la continuité, propriété de l'espace, ce sont deux univers différents qui se construisent. Mais à un certain espace, on peut faire correspondre un certain nombre, qui le mesure, en tant que circonférence ou en tant que surface. Et l'inverse n'est pas moins vrai, du moins dans les cas où les nombres ne sont pas incompatibles avec une part d'expression de la continuité.

On voit sur cet exemple qu'un certain type de relations entre figures peut être commandé par la ressemblance, par imitation, suggérait Pascal. Certes, la ressemblance observée entre géométrie et arithmétique est trop générale pour pouvoir s'appliquer utilement à de vastes domaines du savoir. Mais on peut lui trouver des applications à propos de multiples problèmes particuliers, ceux notamment qui exigent le recours aux quatre opérations. Il en va de même pour d'autres ressemblances simples entre mathématiques et philosophie, que nous devons négliger, pour nous concentrer sur des cas complexes.

On peut toutefois remarquer déjà une de ces applications, toujours commandée par la ressemblance, mais où la philosophie, voire la théologie, ont leur rôle à jouer. Voici ce que Pascal écrit :

Tout ce qui est incompréhensible ne laisse pas d'être: le nombre infini ; un espace infini, égal au fini.

De nouveau, arithmétique et géométrie se trouvent mis en correspondance, et comme en situation de figure l'un de l'autre. Voilà le point essentiel que Pascal voulait faire valoir, en renforçant mutuellement le témoignage de l'arithmétique et celui de la 
géométrie. Mais les deux sciences sont invoquées à cause du rapport qui peut se faire d'elles à la philosophie. Car celle-ci, ou plutôt la théologie, se trouve en face d'une certaine « incompréhensibilité » dans l'étude du rapport entre l'homme et Dieu et, plus précisément, entre la justice divine, qui est infinie, et la justice humaine, égale au fini, c'est-à-dire proche du néant. La question est soulevée par le problème théologique de la justification et du salut. Le refus éventuel du divin résultant de l'incompréhensibilité qu'il comporte est combattu par des exemples mathématiques de la même incompréhensibilité. Ainsi une esquisse de réponse à une objection antireligieuse estelle conduite en définitive à la lumière d'une proportion mathématique.

Les intentions religieuses de Pascal et la force probante qu'il attend pour les remplir du recours aux mathématiques sont indiscutables. Il pourrait nous suffire d'en faire le constat, comme nous l'avons fait, avec justification, lors de la première mise en parallèle de la géométrie et de l'arithmétique. Mais un commentaire un peu plus subtil est ici nécessaire, parce que l'usage fait des mathématiques le requiert. Nous nous garderons de nous laisser entraîner trop longuement hors de notre vrai sujet. Nous indiquerons simplement les difficultés rencontrées, qui n'échappaient sûrement pas à Pascal. Elles touchent à la notion d'infini, et aux deux exemples d'incompréhensibilité qu'elle fournit :

Le nombre infini. Un espace infini, égal au fini.

Le paradoxe concernant l'espace n'est pas le plus déconcertant. La contradiction posée est affirmée imperturbablement. Des multitudes d'exemples en ont été repérées par Dominique Descotes. Le sens commun ne voit pas de difficulté à l'élargissement indéfini d'un espace. Peut-être faudrait-il seulement distinguer entre infini absolu et infini relatif. Pour saisir aussi une contradiction au sujet du "nombre infini", il faut se reporter à une autre pensée de Pascal, développement sans doute de la précédente :

Nous connaissons qu'il y a un infini et ignorons sa nature. Comme nous savons qu'il est faux que les nombres soient finis - donc il est vrai qu'il y a un infini en nombre, mais nous ne savons pas ce qu'il est. Il est faux qu'il soit pair, il est faux qu'il soit impair ; car en ajoutant l'unité, il ne change point de nature. Cependant c'est un nombre et tout nombre est pair ou impair. Il est vrai que cela s'entend de tout nombre fini.

On voit que l'arithmétique est, en définitive, plus complexe que la géométrie. La notion de nombre infini est plus délicate à manier que celle d'espace infini, ou, en d'autres termes, le paradoxe est plus radical avec les nombres qu'avec l'espace.

Quelles difficultés soulève donc l'expression nombre infini ? S'il est facile d'admettre que la série des nombres puisse être indéfiniment prolongée, il l'est beaucoup moins de supposer que cette série s'achève par un dernier nombre, porteur de tous les caractères de l'infinité. Car, alors, ce nombre impossible à prolonger manquerait d'une des propriétés constitutives de l'infinité. Il ne serait pas un nombre. Si Pascal emploie l'expression nombre infini, c'est donc en attribuant à ce nombre une nature spéciale et, s'il est possible de lui prêter cette audace, en en faisant un pur concept. Lequel préserve l'idée d'un nombre, totalement irrationnel, susceptible d'arrêter la prolongation d'une série théoriquement illimitée, parallèlement à celle d'une possibilité permanente, mais impraticable jusqu'au bout, d'accroissement des nombres. Les problèmes ainsi soulevés par l'idée d'infini se retrouvent à propos de celle de zéro, envisagée par Pascal luimême dans son écrit De l'Esprit géométrique. Encore une correspondance entre deux 
objets appartenant à la fois à l'arithmétique et à la géométrie, et offrant une évidente portée générale.

\section{Géométrie et finesse}

Nous demeurons dans la catégorie des figures semblables, en dépit de certaines apparences, si nous envisageons le rapport entre deux expressions fortement marquées du sceau de Pascal, celles d'esprit de géométrie et d'esprit de finesse. Il nous est arrivé souvent déjà de présenter sur ce sujet des réflexions proposées par un pascalien éminent et méconnu, Jean Prigent, et qui allaient heureusement à contre-sens de l'exégèse la plus couramment répandue. Cette dernière consistait à établir une opposition radicale entre les deux types d'esprits distingués par Pascal. L'esprit de géométrie aurait été déductif, et l'esprit de finesse, intuitif, ce qui l'aurait rapproché d'une autre notion chère au même auteur, et qu'il appelle cœur. Une lecture superficielle du texte pouvait sembler autoriser cette interprétation. Il est dit en effet que l'esprit de géométrie use de principes «palpables, mais éloignés de l'usage commun", de sorte qu'on a peine à les aborder, mais qu'en le considérant attentivement, on les voit «à plein » et qu'il est impossible de mal raisonner avec eux. $\mathrm{Au}$ contraire, «dans l'esprit de finesse, les principes sont dans l'usage commun et devant les yeux de tout le monde... Il n'est question que d'avoir bonne vue...; car les principes sont si déliés et en si grand nombre qu'il est presque impossible qu'il n'en échappe. Or l'omission d'un principe mène à l'erreur... » Plus loin, il est dit des « choses de finesse » - et ce passage a été la principale source de méprises sur le sens du propos :

On les voit à peine, on les sent plutôt qu'on ne les voit. Ce sont choses si délicates, et si nombreuses, qu'il faut un sens bien délicat et bien net pour les sentir et juger droit et juste, selon ce sentiment, sans pouvoir le plus souvent le démontrer par ordre comme en géométrie... Il faut tout d'un coup voir la chose d'un seul regard, et non pas par progrès de raisonnement, au moins jusqu'à un certain degré... Ce n'est pas que l'esprit ne le fasse, mais il le fait tacitement, naturellement et sans art...

Il est clair, par ces passages et par leur contexte, que l'esprit de géométrie est celui qui construit la science géométrique, avec comme première propriété de poser des principes et d'en déduire des conséquences. Il est déductif, et possède tous les caractères qui ont été précédemment analysés quant à son fonctionnement et qui le rendent étranger à l'expérience humaine pratique, sans l'empêcher de fournir un excellent moyen de connaissance. De son côté, l'esprit de finesse n'est pas moins déductif, ni moins tributaire de principes commandant le raisonnement. Il a donc la même structure que l'esprit de géométrie : c'est une sorte de géométrie supérieure. Il pourrait, dans l'idéal, fonctionner de la même façon. Ce qui l'en empêche, quoiqu'il s'appuie aussi sur des principes, c'est la nature particulière de ceux-ci. Ils sont à la fois très communs et très nombreux. Les poser un à un et les rappeler tout au long du raisonnement, de même que les embrasser tous à la fois pour assurer la validité de l'argumentation est pratiquement impossible. C'est bien ce qui se constate dans la conduite de l'expérience personnelle comme dans la connaissance générale des choses humaines, sujets constants de la réflexion individuelle et des échanges sociaux. Il faut donc concevoir une déduction compréhensive et assouplie, inscrite dans un espace plus ouvert et dans un temps plus rapide, permettant de sauter certaines étapes intermédiaires du raisonnement en les effectuant de façon tacite. C'est là l'œuvre de cette faculté que Pascal appelle sentiment, c'est-à-dire ni raison, ni intuition, mais sorte 
de perception aiguë et de vive intelligence, que les modernes associeraient peut-être au jeu de l'inconscient.

Quoi qu'il en soit, esprit de géométrie et esprit de finesse, dans leur unité de structure et dans leurs similitudes de fonctionnement, se figurent bien l'un l'autre. On aura remarqué que le premier concerne essentiellement la pure géométrie, tandis que le second, particulièrement adapté au domaine humain, où les faits, bien analysés ou bien synthétisés, peuvent devenir principes, est parfaitement susceptible de s'appliquer à la philosophie.

29 Nous nous en tiendrons là pour l'évocation de certains types de ressemblance entre géométrie, voire mathématiques en général, et philosophie. Nous constaterons cependant beaucoup de faits de même nature, intervenant à titre complémentaire, à propos d'autres situations qu'il nous appartient maintenant de considérer.

\section{Les séries en mathématiques}

Une de ces situations est constituée par les problèmes de séries. Ils apparaissent notamment en arithmétique, ou en théorie des nombres. Pascal les connaît bien. Ils concernent des suites de nombres reliés par un principe d'organisation défini au préalable, nombres dont il s'agit, le plus souvent, de trouver la somme. Les nombres dits naturels sont évidemment déjà une suite de nombres. Mais, à eux seuls, ils ne prêtent pas à des réflexions très originales. Il est en revanche des séries de complexité variable, parfois très grande. En 1654, peu de temps avant la rédaction du Traité du triangle arithmétique, où il s'y applique encore, Pascal a consacré à certaines de ces dernières un remarquable ensemble de petits ouvrages. Parmi les séries ainsi envisagées, beaucoup de recherches restaient à faire sur les puissances, obtenues par multiplication des nombres par eux-mêmes, et sur leurs propriétés, notamment les sommes qui se formaient par leurs successions, totales ou partielles, et quel que soient et le nombre retenu pour commencer, et le nombre générateur de la série. Il y consacra un brillant traité, Potestatum numericarum summa, Sommation des puissances numériques, dépassant de beaucoup les résultats obtenus par les anciens. Sujet arithmétique, sans doute, mais touchant aussi à la géométrie par la considération des carrés et des cubes, sans oublier toutes les puissances de degrés supérieurs, impossibles à figurer, mais non à concevoir. Il en va de même, quoique autrement, pour d'autres modèles de séries qui intéressèrent plus encore Pascal, notamment celle des nombres figurés, entre lesquels ceux dits triangulaires - les mots, on le voit, font encore songer à la géométrie. Ces derniers nombres, obtenus par simple addition de nombres naturels consécutifs, et toujours à partir d'un nombre générateur choisi arbitrairement, forment de nouveau séries ; ils sont nommés, non pas puissances, mais nombres figurés et possèdent encore certaines propriétés. Ils entrent dans ce que Pascal appelle aussi, et de plus en plus volontiers, ordres numériques, et qui, disposés géométriquement, constitueront la figure du triangle arithmétique. D'innombrables problèmes, la solution se trouvait grandement facilitée.

31 Pour rester fidèle à la méthode que nous avons suivie jusqu'ici, nous ne nous élèverons pas jusqu'à la philosophie sans un passage par la géométrie, plus complet que celui qui vient d'être esquissé. La tâche est d'ailleurs relativement aisée. Il suffit de dégager les articulations de la méthode suivie en arithmétique et de chercher quelles correspondances peuvent leur être trouvées en géométrie. 
32 Essayons donc de décrire le schéma sous-jacent aux démarches analysées en arithmétique. Il comporte, chaque fois, deux éléments: l'un, matériel, une série de nombres, l'autre, formel, que nous appellerons le principe de génération de chaque série. Le premier peut se résoudre en une figure, soit ligne marquant progression, soit tableau à usage pratique, comme le dessin du triangle arithmétique. Le second n'est pas susceptible de représentation, mais il est une sorte de clef de la figure, dont tout le sens dérive de lui. Ces deux éléments sont évidemment susceptibles de multiples variations, porteuses d'effets réciproques.

L'œuvre de Pascal fournit plusieurs transpositions géométriques de ce schéma. Elle permet même de passer par transitions insensibles de la géométrie pure à des applications philosophiques.

Dans un cas comme dans l'autre, une remarque fondamentale s'impose. Passer de l'abstraction arithmétique à l'image géométrique peut impliquer l'entrée en scène d'un observateur, dont le lieu de station et l'orientation de regard se composent dans le principe de génération dont va dériver la figure.

Prenons l'exemple des coniques. Il est d'autant plus intéressant que, dans l'œuvre que Pascal leur a consacrée, en grande partie perdue, subsiste un fragment essentiel, copié par Leibniz, sorte de chapitre introductif, dont le titre, Generatio conisectionum, Génération des sections coniques, enferme le mot sur lequel nous avons attiré l'attention. Un mot qu'explique fort bien le texte qui suit, lequel offre un rapport évident avec ceux que nous avons rencontrés concernant les séries de nombres. En effet, le point qu'occupe le sommet du cône, équivalent du chiffre 1 par lequel débutent les plus élémentaires des séries arithmétiques, constitue le lieu où se situe l'œil de l'observateur, et où il peut contempler la génération de toute une série de figures. Représentons-nous un plan perpendiculaire à l'axe du cône et venant couper la surface de ce dernier. Au départ, il rencontrera le sommet du cône et n'y découpera qu'un point, identique à celui qu'occupe l'œil de l'observateur et amorce de la série. En allant vers la base du cône, c'est un cercle qu'il découpera, et même une multitude de cercles, suivant la distance parcourue, mais possédant tous, hors leurs dimensions, les mêmes propriétés. Et il faut poursuivre. Si le plan du cercle s'incline sur l'axe du cône, le cercle lui-même va se transformer en plusieurs figures successives, ellipse, parabole, hyperbole, en somme toutes les sections coniques, ainsi rangées dans une même famille et offrant des possibilités de saisie globale. On pourra dire aussi que l'image du cercle vue du sommet du cône subira plusieurs métamorphoses.

Michel Serres, dans une étude justement fameuse, a fait de ce schéma géométrique la matrice de l'argumentation de Pascal sur la raison des effets. À notre avis, si le principe d'une matrice géométrique doit être conservé, c'est ailleurs qu'il faut le chercher. Nous n'allons pas tarder à le montrer.

Nous retiendrons seulement du présent exemple que la succession dont il rend compte ne s'opère pas d'une manière strictement régulière, comme c'était le cas avec les séries arithmétiques. Il se produit deux mouvements différents, l'un antérieur, l'autre postérieur à l'inclinaison du plan du cercle. En outre, le second mouvement se décompose en plusieurs selon les figures qu'il fait ressortir. Cette présence du mouvement, qui prolonge la géométrie en mécanique, se prête merveilleusement à l'exploitation du raisonnement figuré dans l'étude des problèmes philosophiques ou des relations humaines. 

Pascal, quoiqu'il ait encore laissé peu d'écrits sur le sujet. C'est même surtout dans les Pensées qu'il s'y réfère, accusant ainsi le fait d'un rapport étroit entre les discours tenus par le géomètre et par le philosophe.

pensées sont particulièrement expressives à cet égard. On remarquera qu'elles adoptent très peu le vocabulaire géométrique, marquant seulement en termes concrets la différence entre le grand et le petit selon la distance entre l'observateur et l'objet. La plus pittoresque évoque les relations entre Pascal et l'un de ses maîtres en mathématiques, Desargues, qui possédait des vignobles réputés dans la vallée du Rhône, à Condrieu :

On distingue des fruits les raisins, et entre ceux-là les muscats, et puis Condrieu, et puis Desargues, et puis cette ente. Est-ce tout? En a-t-elle produit deux grappes pareilles ? et cette grappe a-t-elle deux grains pareils ? etc.

Le regard s'étend du général au particulier, plus exactement de l'ensemble au détail, suivant sans doute la voie d'une découverte progressive à l'arrivée sur les lieux. Au-delà du pittoresque et de la variété des objets qui se présentent successivement, c'est l'idée de la diversité du monde qui est mise en avant, avec celle de la difficulté pour l'homme de se situer dans cette multiplicité, en somme de juger.

41 Un commentaire semblable, appelant quand même quelques réflexions nouvelles, peut s'appliquer à la seconde pensée :

Une ville, une campagne, de loin est une ville et une campagne, mais à mesure qu'on s'approche, ce sont des maisons, des arbres, des tuiles, des feuilles, des herbes, des fourmis, des jambes de fourmis, à l'infini. Tout s'enveloppe sous le nom de campagne.

La diversité des objets atteints est mieux marquée que dans la précédente pensée, et soulignée par l'ampleur du vocabulaire. Comme, si la ville et la campagne, selon qu'elles sont vues de loin ou de près, changeaient de nature. Pascal soulève au fond là le vieux problème des universaux. Mais, en le rattachant à la perspective, il le renouvelle considérablement.

43

Tous les faits de succession, dans un cadre géométrique, auxquels nous ont conduits les précédentes analyses ne sauraient trouver meilleur couronnement que dans l'une des plus célèbres pensées, celle qui est intitulée Disproportion de l'homme, et qui trace l'image saisissante que l'on sait de l'homme perdu dans l'univers, écartelé entre l'infiniment grand et l'infiniment petit. Elle présente à la fois une solide armature géométrique et une grande force philosophique, qui reçoivent l'une et l'autre le vêtement d'une haute qualité littéraire. Si la géométrie y joue un rôle particulier, c'est d'abord à cause de la structure générale du texte. Tant dans la montée vers l'infiniment grand que dans la descente vers l'infiniment petit, nous parcourons une série de cercles concentriques, dessinés par l'œil de l'observateur, placé sur terre et explorant les espaces successifs qu'il découvre. Espaces grandissants d'abord, fournis par la sensation, qui embrasse la terre, puis par la science, qui saisit l'orbite du soleil, puis le parcours des astres, à continuer par les espaces imaginables, et par ceux qui ne le sont pas. Grandissement signifié d'ailleurs moins par les hyperboles que par les notations de petitesse appliquées à tout ce qui n'est pas de la grandeur supérieure. L'exploration de l'infiniment petit se fait davantage par recours à l'expérience concrète. Mais l'imagination y apparaît aussi, d'une autre manière; de même que le recours à des

Courrier du Centre international Blaise Pascal, 33 | 2011 
antithèses signifiant le contraire de ce qu'elles disent, comme celle qui fait voir dans le plus petit objet possible « une infinité d'univers ».

Texte qui prend tout son sens lorsqu'il est mis en rapport avec un autre fragment célèbre qui lui fait suite, celui qui dépeint l'homme comme un "roseau pensant », et ainsi, en quelque façon, maître, par son choix de vie, de cet univers qui l'écrase. La philosophie doit quelquefois dépasser cette géométrie qui la guide si souvent.

Il vaudrait la peine de chercher plus longuement dans les Pensées des traces de cette exploitation par Pascal de la réflexion arithmétique et géométrique sur les séries. Elle aboutit en particulier à la mise en évidence de la notion de modèle, remarquablement perçue par Pascal. C'est ce qu'a fort bien montré Louis Marin, en s'appuyant, comme il convenait, sur les fragments relatifs à la Beauté poétique. On se contentera ici de rappeler la phrase principale, en la rattachant brièvement à notre propos :

Il y a un certain modèle d'agrément et de beauté qui consiste en un certain rapport

entre notre nature, faible ou forte, telle qu'elle est, et la chose qui nous plait.

En d'autres termes, la beauté tient à la constitution de l'homme, de chaque homme, et se définit par un rapport de convenance de telle personne, ou de telle catégorie de personnes, avec l'objet jugé beau. Dès lors des modèles se trouvent définis, semblables aux principes organisateurs des séries, et exprimés par des rapports. De nouveaux types de séries se constituent ainsi, comprenant toutes les personnes qui ont les mêmes " goûts ", et surtout les divers objets qui répondent au modèle. Entre ces derniers se dessinent des " correspondances », au sens baudelairien du terme, facteur d'unité dans l'univers. Ainsi la science et l'art se rejoignent.

\section{Les combinaisons}

47 À la suite des séries, si nous reprenons nos repères arithmétiques, se découvrent les combinaisons, objet aussi d'une vive attention au cours de l'année 1654 de la part de Pascal, comme de celle de Fermat. La combinatoire n'envisage plus de créer des successions planifiées, finies ou infinies. Elle joue sur des totalités et s'efforce d'établir des relations entre celles-ci et les parties, en quelque nombre que ce soit, qui les composent ou peuvent les composer.

Si la notion de combinaison s'applique surtout à l'arithmétique, nous nous permettrons quand même d'en élargir le sens, et de lui faire désigner des figures complexes, ou formées par composition de figures, dès lors qu'elles reposent sur un fondement géométrique et que leur rapport avec la construction philosophique est indiscutable.

Nous analyserons deux exemples, le premier, concernant les trois ordres de grandeur, déjà sommairement reconnu en 1922 par Jacques Chevalier, le second, éclairant d'une lumière vive le raisonnement par la raison des effets, entièrement inédit.

Revenons donc au traité Potestatum numericarum summa. Quel objet Pascal y poursuit-il exactement?

51 Comme le titre l'indique, il s'agit de proposer une méthode permettant de calculer la somme d'une série de puissances numériques. Mais la donnée du problème est certainement un peu plus précise. En effet la recherche s'applique à un sujet aussi large que difficile, mais très exactement défini. Elle porte sur ces séries de nombres que l'on appelle progressions arithmétiques, et dont le principe, déjà évoqué précédemment, indique pour chacune d'elles le premier et le dernier nombre de la série, tandis qu'un 
autre nombre marque la "différence ", dit Pascal, à quoi nous substituerions volontiers « l'intervalle » qui sépare les nombres en progression. Aussi bien, si nous écrivons 4, 7, 10,13 , nous savons quels sont le premier et le dernier nombre en progression, ainsi que leur « différence », c'est-à-dire 3.

Mais l'objet de Pascal n'est pas encore suffisamment caractérisé. Ce qu'il convient de calculer, ce n'est pas la somme de certains nombres, mais celle de leurs puissances, envisagées par progression, selon leurs degrés naturels et dans des limites préalablement tracées, enfin énoncées successivement. C'est de tout cet ensemble qu'il conviendra de faire la somme.

Ce qui ressort au terme de cette enquête redoutable, c'est qu'une des lignes ainsi superposées, celle qui porte les « racines ", c'est-à-dire les nombres simples, points de départ, mais non puissances, n'a pas à entrer dans le calcul, dont elle est éliminée. Comme il est assez naturel, les puissances ne commencent qu'avec le second degré, les carrés (exposant 2). Dans la pratique, Pascal ne les poursuit guère qu'avec les cubes (exposant 3) et les carrés-carrés (exposant 4), mais théoriquement, il sait fort bien qu'ils peuvent se prolonger indéfiniment. Ce qui se dit des racines par rapport à la puissance 2 peut d'ailleurs se dire de cette dernière par rapport à la puissance 3 , et ainsi de suite.

Du tout est tirée rapidement la conclusion :

Les racines ne comptent pas rapport aux carrés, les carrés par rapport aux cubes,

les cubes par rapport aux carrés-carrés, etc.

Mais l'auteur de cette phrase avait déjà, dans le même paragraphe, et selon sans doute un plan préétabli, élargi sa réflexion par une application à la géométrie qui lui tenait beaucoup plus à cœur. Voici ses termes, traduits du latin :

Dans le cas d'une grandeur continue, des grandeurs d'un genre quelconque, ajoutées, en tel nombre qu'on voudra, à une grandeur d'un genre supérieur, ne l'augmentent de rien. Ainsi les points n'ajoutent rien aux lignes, les lignes aux surfaces, les surfaces aux solides...

Dans ces lignes, le mot « genre » a exactement le même sens que celui d'« ordre », plus usuel dans les Pensées. Nous venons en effet de saisir le fondement géométrique, et schématique, du célèbre fragment sur les trois ordres de grandeur. Celui-ci est pourtant d'ordre strictement et profondément philosophique. Des relations typiquement géométriques ont été parfaitement préservées. Mais, comme on le trouvera aussi chez Leibniz, des concepts sont venus prendre la place des objets mathématiques, lesquels leur auront procuré, de leur côté, une précision et une solidité dont la philosophie ne saurait faire fi.

57 Sans reprendre le commentaire de cet éblouissant fragment, auquel nous nous sommes déjà longuement appliqué, nous insisterons plus que nous ne l'avons encore fait, sur son rapport avec ses origines géométriques.

58 Nous développerons d'abord brièvement la remarque déjà faite plus haut sur les combinaisons qui s'y opèrent entre plusieurs types de figures. Le plus important, comme il résulte des analyses déjà faites, consiste dans la mise en œuvre brillante du modèle de la série ou progression, avec passage méthodique, et multiforme, de l'arithmétique à la géométrie, et achèvement dans la philosophie. De même, la progression entre grandeurs depuis les plus basses jusqu'aux plus hautes n'est pas moins cohérente mathématiquement que philosophiquement. Il y a lieu d'observer aussi une autre exactitude mathématique. L'annulation des valeurs de l'ordre inférieur 
devant celles de l'ordre supérieur se réalise de manière effective chaque fois qu'un passage se produit, mais uniquement dans le sens de la montée. Aussi bien, l'accession à l'ordre du cœur annule-t-elle les deux ordres précédents. Mais, en sens inverse, l'ordre des esprits ne perd pas ses ressources lorsqu'il en fait bénéficier l'ordre des corps, ni l'ordre du cœur lorsqu'il s'incline vers les deux premiers ordres. On remarquera aussi la discrétion relative avec laquelle est évoquée la notion d'infini, tant en géométrie qu'en philosophie. Certes les ordres sont infinis dans leur constitution. Mais leur infinitude est ordonnée, ou, si l'on préfère, leurs infinitudes sont subordonnées l'une par rapport à l'autre - ce qui, d'ailleurs ne contredit nullement les mathématiques. C'est même pour cette raison qu'il peut y avoir des degrés d'infinités et des infinis d'infinis Toutefois, les ordres sont en nombre limité. S'ils sont trois, c'est sans doute à cause des trois dimensions de l'espace (du moins l'espace perçu par l'homme): nouveau salut à la géométrie.

Un autre type de figures se dessine aussi dans ce fragment : celui que caractérise le jeu sur la ressemblance que suggère d'abord le mot «figure ». Car au souci de mettre en valeur des contrastes s'ajoute un sens remarquable des proportions et des enseignements qu'elles offrent. Chaque ordre est proportionné aux autres. À chacun répond une certaine grandeur, signifiée par le retour du mot lui-même, mais aussi par tous les synonymes et toutes les images qui en renforcent l'idée. Pascal invente luimême une proportion inédite et grandiose, parfaitement géométrique, mais non moins solide sous l'angle de la rhétorique et de la philosophie, lorsqu'il évoque la distance des corps aux esprits, et la compare à celle, "infiniment plus infinie, des esprits à la charité ». Ainsi se combinent les deux types de figures.

On reconnaît que ce jeu de combinaisons ôte beaucoup de leur force sensible à des figures certes présentes derrière la surface du texte, mais dont les traits se sont quelque peu estompés sous la force intellectuelle des idées. Mais c'est un trait constant de l'écriture de Pascal, qui fait un grand usage des images, que de les présenter sous forme d'esquisses ou d'ébauches, voire de les estomper comme nous venons de le constater. Leur présence n'en est que plus efficace, car elle prépare à l'idée et soutient la réflexion sans s'y substituer.

\section{Raison des effets et figure géométrique}

61 L'harmonie et la puissance de ce fragment nous ont offert beaucoup de moyens pour en conduire avec exactitude l'analyse et le commentaire. La perfection formelle du texte nous y aidait aussi, preuve de l'extrême attention avec laquelle l'auteur l'avait composé. Enfin l'absence de brouillons préparatoires garantissait un écrit achevé. Aucun des avantages ainsi procurés n'était disponible pour aborder le thème de la Raison des effets. L'importance du sujet ne pouvait cependant faire de doute, puisque l'expression servit de titre à l'un des premiers chapitres, et des plus longs, des Pensées, et qu'elle reparaissait dans plusieurs autres fragments. Mais peu d'entre eux offraient un caractère très élaboré, et, s'ils se complétaient souvent, il était parfois assez difficile de les agencer. Un certain rapport était assez facile à établir avec un autre titre du même chapitre, Opinions du peuple saines, qui avait été choisi d'abord, pour être ensuite mis de côté. Mais comment interpréter ce rapport, et pourquoi ce changement ? L'hésitation était de mise. Elle a certainement été le fait des premiers éditeurs des 
Pensées lorsqu'ils ont découvert les fragments inachevés: ils ont négligé un grand nombre de ceux-ci.

\section{GRADATION}

Le peuple honore les personnes de grande naissance. Les demi-habiles les méprisent, disant que la naissance n'est pas un avantage de la personne, mais du hasard. Les habiles les honorent, non par la pensée du peuple, mais par la pensée de derrière. Les dévots, qui ont plus de zèle que de science, les méprisent, malgré cette considération qui les fait honorer par les habiles, parce qu'ils en jugent par une nouvelle lumière que la piété leur donne. Mais les chrétiens parfaits les honorent par une autre lumière supérieure.

Ainsi se vont les opinions succédant du pour au contre selon qu'on a de lumière.

Il serait possible d'ajouter d'autres articulations à ce raisonnement. Mais elles n'ajouteraient rien au schéma fondamental, qui doit seul nous retenir.

On notera d'abord qu'il s'agit d'un fragment relatif à l'homme, ou à la société, et aux attitudes qui s'y rencontrent, celles-ci décrites comme des faits et non comme des règles à suivre, même si elles font l'objet de jugements de valeur. C'est le moraliste, au sens ancien du terme, qui parle, et non le guide religieux. Le savoir recherché est d'ordre quasi scientifique. Mais il adopte d'autres moyens que ceux des sciences exactes. Il procède par des sortes d'approximations, conduisant à passer par des attitudes extrêmes et contraires pour recommencer en revenant toujours, sinon au point de départ, du moins à une situation de même type.

Michel Serres comme nous l'avons déjà noté, a proposé une très brillante exégèse de ce texte en cherchant l'origine du raisonnement dans celui dont découle la génération des sections coniques. Il a cependant relevé quelques difficultés dans cette théorie, qui, à notre sens, obligent à l'écarter.

Pour formuler une autre hypothèse, il nous faut d'abord, comme nous en avons déjà donné l'exemple, reconstituer le schéma du raisonnement. Il est d'ordre incontestablement géométrique, ou plutôt, peut-être, mécanique, parce qu'il donne grande part au mouvement; mais il n'y a pas là de différence essentielle. Il envisage deux mouvements simultanés, ce qui peut constituer l'une de ces combinaisons de figures auxquelles nous entendons nous appliquer. L'un de ces mouvements est d'ordre circulaire. Il est porté par un cercle destiné à rouler dans une direction rectiligne occupant une position de tangente et qui commandera un second mouvement. Au départ, le cercle s'appuie sur un premier point de tangence, début de son mouvement à venir, qui sera aussi le début de l'itinéraire rectiligne déterminé par ce mouvement. Le même point joue en même temps le rôle de signe. Il représente la position du peuple, que Michel Serres aurait désignée par la réponse oui. Le point ainsi marqué étant arrivé, après un demi-tour, au sommet du cercle, il change de signification et est occupé par le demi-habile, qui dit non. Un autre demi-tour et le cercle, après translation complète, reprend sa disposition initiale et le oui revient en bas. Le cercle continue ensuite à tourner et la même succession reparaît avec les dévots et les chrétiens parfaits, les deux extrémités d'un diamètre, opposées géométriquement, ne l'étant pas moins philosophiquement. On pourrait continuer en imaginant d'autres types d'attitudes.

Courrier du Centre international Blaise Pascal, 33 | 2011 
Cependant, malgré le retour des positions contraires, et le fait même des contradictions, le temps du trajet n'a pas été perdu parce que les étapes opposées auront été propices à la réflexion et à la prise en compte de " pensées de derrière ».

pproximations progressives de vérité auront ainsi été obtenues, signifiées par le parcours rectiligne, image naturelle du progrès, réalisé par le cercle « rectifié », alors que le cercle fermé signifie l'éternel retour.

69 On a reconnu quelle était la figure géométrique présente à l'esprit de Pascal lorsqu'il tenait ce raisonnement particulièrement original et subtil. C'est tout simplement celle que dessine le point de départ du double mouvement lorsque le cercle accomplit un tour complet en roulant sur la ligne droite, en somme, celle de la fameuse roulette ou cycloïde, suggérée concrètement par le mouvement d'un clou fiché dans la roue d'une charrette en marche, courbe affectant la forme d'une sorte de cloche, et totalement irrationnelle, c'est-à-dire sans équation. Pascal en a dénombré de précieuses propriétés au moment même où il composait les Pensées.

Reste à savoir pourquoi cette figure l'a ainsi inspiré, et quelles affinités existaient entre elle et les idées qu'elle pouvait appuyer. Il nous semble surtout qu'elle nous montre en Pascal un esprit beaucoup moins dogmatique qu'on ne le croit communément, beaucoup plus ouvert au dialogue et à la discussion organisée.

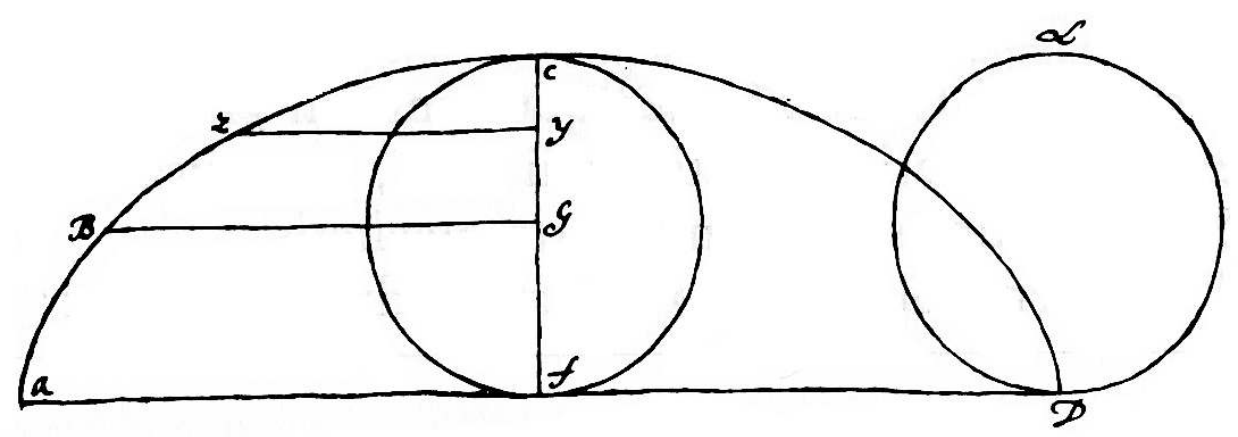

Voilà une moisson dont l'abondance peut surprendre. Quelques questions se posent au moment de conclure.

71 Tout d'abord, avons-nous épuisé la série des cas où l'analyse des rapports que nous avons essayé de saisir peut se révéler efficace? Il est certainement possible de la prolonger : le champ est ouvert à d'autres recherches. Toutefois on contestera peutêtre d'emblée l'absence d'un des fragments les plus célèbres des Pensées, et le plus évidemment nourri de culture mathématique, au point de pouvoir passer lui-même pour une illustration de cette science. On a reconnu le pari. Nous aurions évidemment aimé l'exploiter pour préciser l'analyse de la réflexion portée par Pascal sur la notion d'infini. Si nous l'avons écarté, c'est parce qu'il ne correspondait pas exactement à la nature de l'enquête conduite ici. Nous serions passés de la géométrie au calcul des probabilités, et, plus précisément, à la théorie des jeux. Il est certain que d'amples résultats pouvaient être aussi attendus dans ce nouveau domaine. Délibérément, le champ des mathématiques n'a pas été entièrement couvert. Voilà d'autres voies à explorer. 

cette application de la géométrie à la philosophie ? S'agit-il, non pas d'un simple jeu, mais d'une démarche de type métaphorique, s'attachant à des ressemblances de caractère accessoire, sans engager de relation essentielle? La difficulté est beaucoup plus complexe qu'on ne pourrait le croire. Elle se situe aux frontières de la linguistique et de la métaphysique. Réservons-la pour le moment comme matière à débat.

Il nous semble pourtant qu'au moins pour Pascal, la relation établie avait une signification profonde. Avec elle, nous atteignons un philosophe adepte convaincu d'une Mathesis universalis, dont la nécessité était posée depuis Galilée proclamant que la nature parle le langage des mathématiques, et que plusieurs mathématiciens de son temps s'efforçaient de construire, chacun à sa façon. Ce mouvement trouvera son champion en la personne de Leibniz. Il nous semble avoir donné la preuve que la pensée la plus générale de Pascal s'y inscrit aussi en profondeur. Enfin, avec la référence géométrique, pourvoyeuse d'innombrables modes de raisonnement, c'est toute la logique traditionnelle qui se trouve dépassée. Des modèles nouveaux se proposent, applicables à des objets beaucoup plus divers, et exploitant une rationalité elle-même diverse, où les grands principes sont susceptibles de retouches bénéfiques pour l'accession à une vérité plus souple, plus conforme à la nature des choses et à celle de l'homme. La religion elle-même en devient plus familière. S'il en est bien ainsi, la présence de Pascal dans la philosophie moderne s'élargira considérablement.

\section{BIBLIOGRAPHIE}

Une première esquisse de cette étude a été présentée au séminaire de Michel Serfati, à l'Institut Henri Poincaré de Paris, le 9 mars 2011.

L'orientation de la recherche conduite a été partiellement suggérée par deux ouvrages majeurs, celui de Jacques Chevalier (s'inspirant d'ailleurs déjà de Fortunat Strowski, Pascal et son temps, t. II, Paris, Plon, 1907, p. 288-292), dans son Pascal, Paris, Plon, 1922, p. 180-185, 321-324 ; et celui de Michel Serres, Le système de Leibniz et ses modèles mathématiques, t. II, Paris, PUF, 1968, (chapitre Le paradigme pascalien, p. 647-712).

Les passages relatifs au statut de la géométrie selon Pascal se retrouvent dans leur contexte si l'on se reporte à notre édition de Pascal, CEuvres complètes, t. I, Paris, Desclée De Brouwer, 1964, p. 573, 605 ; t. III, 1991, p. 429-437 ; t. IV, 1992, p. 923.

Sur les concordances entre disciplines mathématiques différentes, nous utilisons les Pensées, successivement S 577, LG 592 (S désignant l'édition de Philippe Sellier, Paris, Garnier, 1981 (1 ${ }^{\text {re }}$ éd., Mercure de France, 1976), LG celle de Michel Le Guern, Paris, Gallimard, 1977) ; S 182, LG 139 (avec le brillant commentaire apporté par Dominique Descotes) ; S 680, LG 397.

Sur géométrie et finesse, S 670, LG 466. 
Sur le triangle arithmétique et les traités connexes, voir notre édition parmi les Euvres complètes, t. II, 1970, p. 1166-1332 (pour Potestatum numericarum summa, p. 1259-1272).

Sur la Generatio conisectionum et ses diverses exploitations, voir ibid., p. 1102-1131. Nos réflexions touchant la perspective sont appuyées sur S 465, LG 479 ; S 99, LG 61 ; S 230, LG 185.

Pour les fragments sur la « beauté poétique », S 486, LG 500. Voir l'article de Louis Marin, « Réflexions sur la notion de modèle chez Pascal », Revue de Métaphysique et de Morale, 1967, p. 89-108.

Sur les trois ordres de grandeur, nous nous permettons de renvoyer à notre article, qui se trouve ici complété, « Le thème des trois ordres dans l'organisation des Pensées », [communication au Colloque de London, Ontario, Canada, en avril 1984], Pascal, thématique des Pensées, Paris, Vrin, 1988, p. 29-55, repris dans La Culture du XVIIe siècle, Paris, PUF, 1992, p. 462-484.

Pour le fragment cité sur la raison des effets S 124, LG 83. Voir aussi tout le contexte.

Dominique Descotes, « Espaces infinis égaux au fini », in Le grand et le petit, CRDP, ClermontFerrand, 1990, p. 41-67.

\section{INDEX}

Mots-clés : Pascal, géométrie, philosophie

Keywords : Pascal, geometry, philosophy

\section{AUTEUR}

\section{JEAN MESNARD}

Académie des sciences morales et politiques 